\title{
A requiem for a dream - A critical evaluation of the role of genomic research in precision nutrition
}

\author{
Marie-Joe Dib ${ }^{1}$, Ruan Elliott ${ }^{1}$ and Kourosh R Ahmadi ${ }^{1}$ \\ ${ }^{1}$ Department of Nutritional Sciences, School of Biosciences and Medicine, University of Surrey, Guildford GU2 $7 X H$
}

Rapid advances in "omics" technologies have paved the way forward to an era where more "precise" approaches, which leverage data on genetic variability alongside the traditional indices, have been put forth as the state-of-the-art solution to redress the effects of malnutrition across the life course, thus reforming the concept of "personalised" into "precision" nutrition. Multiple initiatives (www.ukbiobank.ac.uk/), (www.genomicsengland.co.uk/) (allofus.nih.gov/) have been initiated and all have the common, overarching goal of providing novel insight(s) into disease aetiology and the promise of a new perceived era of "better tests, better drugs and above all better, more personalised care to save lives" (1). The reactions to these initiatives has been mixed ${ }^{(2,3)}$. We purport that while personalised nutrition is already commonly used in dietetic practice, and largely without use of genetic information, the move to precision nutrition approaches that advocate the use genetic data is premature. To achieve the full scope of precision nutrition, it is imperative to first review and synthesise the existing evidence - or lack thereof - from large-scale genetic epidemiology studies that have aimed to dissect the complex interplay of diet and genomics before we even contemplate their utility to dissect human health disparities.

We systematically reviewed the literature using the GWAS catalogue (https://www.ebi.ac.uk/gwas/) and NCBI to identify all genome-wide association studies (GWAS) (2005-18) in relation to micronutrient disposition including biomarkers of micronutrient status and function. From all included studies we extracted summary data on heritability and identified variants explaining heritability in Caucasians.

Focusing on GWAS of micronutrient disposition, we found that a large number of loci associated with static and functional indices of micronutrient status have been identified. Mean estimates of heritability of micronutrient status ranged between $20-35 \%$ for minerals, 56-59\% for water-soluble and 30-70\% for fat-soluble vitamins. With some notable exceptions and in line with findings from GWAS of other common, multifactorial traits, the majority of the identified genetic variants explained little of the overall variance in status for each micronutrient, ranging between $1.3-8 \%$ for minerals, $<0.1-12 \%$ for water-soluble vitamins and $1.7-2.3 \%$ for fatsoluble vitamins.

Our findings highlight, however, that GWAS have provided some novel insight into mechanisms that underpin variability in micronutrient status. We also highlight obvious gaps that need to be addressed if the full scope of precision nutrition is ever to be realised, including research aimed at (i) dissecting the genetic basis of micronutrient deficiencies or "response" to intake/supplementation (ii) identifying trans-ethnic and ethnic-specific effects (iii) identifying gene-nutrient interactions for the purpose of unravelling molecular 'behaviour' in a range of environmental contexts.

1. Genomics England launched, mapping DNA to better understand cancer, rare and infectious diseases [Internet] (2013) Available from: https://www. genomicsengland.co.uk/genomics-england-launch/.

2. Bayer R, Galea S (2015) N Engl J Med 373(6), 499-501.

3. Khoury MJ, Iademarco MF \& Riley WT (2016) Am J Prev Med 50(3), 398-401. 